\title{
Surfing close to the wind
}

\author{
Astrophys. J. Lett. 874, L15 (2019)
}

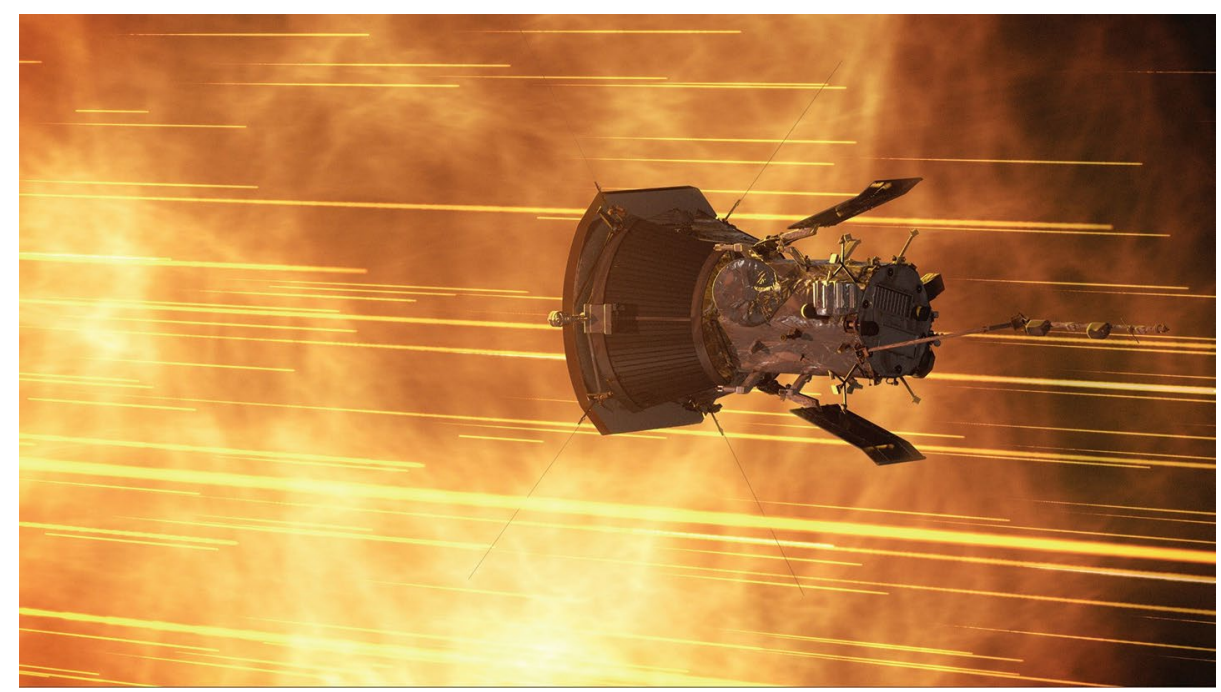

Credit: NASA

On 4 April 2019, the Parker Solar Probe (PSP) made its second perihelion encounter, passing a mere 24.8 million kilometres from the Sun. While waiting for the release of data from the first perihelion made last November, Pete Riley and co-workers ran simulations to predict the structure of the solar corona and inner heliosphere that PSP would have encountered.

The PSP mission will use a series of gravity assists from Venus to make 24 orbits of the Sun, ultimately reaching a closest approach of 6.9 million kilometres at $200 \mathrm{~km} \mathrm{~s}^{-1}$ - a speed comparable to that of the slow solar wind. Given the trajectory and short time window for collecting data, global models of the photospheric magnetic field provide a complementary tool for analysing the data and predicting future coronal states.

Riley et al. applied the

Magnetohydrodynamic Algorithm outside a Sphere (MAS) and found that a week before perihelion, the solar wind about the spacecraft could be traced to an equatorial coronal hole of positive polarity. But then just before perihelion, the connection jumped to a different equatorial coronal hole, to the east, and of negative polarity. After the first close encounter, the spacecraft then became magnetically connected to a northern coronal hole of positive polarity. The authors also used the Potential Field Source Surface (PFSS) model to calculate the coronal magnetic field. There were global similarities, but also differences; in the PFSS simulation, post-perihelion the spacecraft connected to a southern coronal hole. Once the observational data have been analysed, it will be possible to see which model works better, and whether the predicted magnetic field and plasma variables are indeed accurate. This information will hopefully provide insights into coronal heating and solar wind acceleration.

\section{May Chiao}

Published online: 26 April 2019

https://doi.org/10.1038/s41550-019-0783-2 